\title{
META Score: An International Consensus Scoring System on Mesh-Tissue Adhesions
}

\author{
L. C. L. van den Hil ${ }^{1,2}$ - E. H. H. Mommers ${ }^{1,2}$ - J. W. A. M. Bosmans ${ }^{1,2}$ - S. Morales-Conde ${ }^{3}$. \\ V. Gómez-Gil ${ }^{4} \cdot$ K. LeBlanc ${ }^{5} \cdot$ A. Vanlander ${ }^{6} \cdot$ E. Reynvoet $^{6} \cdot$ F. Berrevoet ${ }^{6} \cdot$ S. Gruber-Blum $^{7}$. \\ E. Altinli ${ }^{8}$ - C. R. Deeken ${ }^{9}$ R. H. Fortelny ${ }^{10}$ - J. W. Greve ${ }^{11} \cdot$ K. Chiers $^{12} \cdot$ R. Kaufmann ${ }^{13}$. \\ J. F. Lange ${ }^{13}$ - U. Klinge ${ }^{14} \cdot$ M. Miserez ${ }^{15}$ - A. H. Petter-Puchner ${ }^{7,10} \cdot$ M. H. F. Schreinemacher ${ }^{1,2}$. \\ N. D. Bouvy ${ }^{1,2}$
}

\begin{abstract}
Background Currently, the lack of consensus on postoperative mesh-tissue adhesion scoring leads to incomparable scientific results. The aim of this study was to develop an adhesion score recognized by experts in the field of hernia surgery.

Methods Authors of three or more previously published articles on both mesh-tissue adhesion scores and postoperative adhesions were marked as experts. They were queried on seven items using a modified Delphi method. The items concerned the utility of adhesion scoring models, the appropriateness of macroscopic and microscopic variables, the range and use of composite scores or subscores, adhesion-related complications and follow-up length. This study comprised two questionnaire-based rounds and one consensus meeting.

Results The first round was completed by 23 experts (82\%), the second round by 18 experts $(64 \%)$. Of those 18 experts, ten were able to participate in the final consensus meeting and all approved the final proposal. From a total of 158 items, consensus was reached on 90 items. The amount of mesh surface covered with adhesions, tenacity and thickness of adhesions and organ involvement was concluded to be a minimal set of variables to be communicated separately in each future study on mesh adhesions.

Conclusion The MEsh Tissue Adhesion scoring system is the first consensus-based scoring system with a wide backing of renowned experts and can be used to assess mesh-related adhesions. By including this minimal set of variables in future research interstudy comparability and objectivity can be increased and eventually linked to clinically relevant outcomes.
\end{abstract}

L. C. L. van den Hil

1.vandenhil@maastrichtuniversity.nl

1 Department of General Surgery, Maastricht University Medical Centre, PO Box 5800, 6202 AZ Maastricht, The Netherlands

2 NUTRIM School of Nutrition and Translational Research in Metabolism, Maastricht University, Maastricht, The Netherlands

3 Unit of Innovation and Minimally Invasive Surgery, University Hospital Virgen Del Rocío, Seville, Spain
4 Department of Surgery, Medical and Social Sciences, Faculty of Medicine and Health Sciences, Networking Research Centre on Bioengineering, Biomaterials and Nanomedicine (CIBER-BBN), University of Alcalá, Alcalá de Henares, Madrid, Spain

5 Our Lady of the Lake Physician Group, Minimally Invasive Surgery Institute, Baton Rouge, LA, USA

6 Department of General and Hepatobiliary Surgery, Ghent University Hospital, Ghent, Belgium

7 Ludwig Boltzmann Institute for Experimental and Clinical Traumatology, Austrian Cluster for Tissue Regeneration, Vienna, Austria 


\section{Introduction}

Intra-abdominal mesh placement for incisional hernia repair is a well-known inducer of adhesions between intestines and mesh [1]. Intra-abdominal adhesions are a known cause for serious postoperative complications such as bowel obstruction and abdominal pain $[2,3]$. To prevent such complications, many meshes for intra-abdominal application have been designed using either antiadhesive coatings or different materials such as PTFE to prevent adhesion formation.

Important factors influencing adhesion formation are mesh material, pore size and the presence of antiadhesive coatings [4]. These factors may influence the number of adhesions, though complete adhesion prevention is currently not possible $[4,5]$. Ideally, new designs and concepts could be tested in vitro, but with a lack in understanding and combining of so many variables involved in adhesion formation, testing of new surgical meshes still depends heavily on animal testing [6, 7]. Unfortunately, comparing the efficacy of antiadhesive devices, both clinically and in animal research, has become increasingly difficult due to the abundance of various adhesion scores. Currently, there are over twenty different experimental and clinical adhesion scores available [8-10]. Clearly, there is a lack of consensus on the ideal adhesion scoring method, and interstudy comparability is decreasing due to the increasing diversity in adhesion scores [8, 10-12]. Subsequently, this could lead to redundant tests with less reduction of animals and increased costs.

The goal of this study was to achieve consensus on an adhesion scoring method focusing on mesh-related

8 Department of General Surgery, Bilim University, Istanbul, Turkey

9 Covalent Bio, LLC, St. Louis, MO, USA

10 Department of General Surgery, Wilhelminenspital Der Stadt Wien, Vienna, Austria

11 Department of General Surgery, Zuyderland Medical Center, Heerlen, The Netherlands

12 Department of Veterinary Pathology, Faculty of Veterinary Medicine, University of Ghent, Ghent, Belgium

13 Department of Surgery, Erasmus University Medical Center Rotterdam, Rotterdam, The Netherlands

14 Department of General, Visceral and Transplantation Surgery, RWTH Aachen University Hospital, Aachen, Germany

15 Department of Abdominal Surgery, University Hospitals, KU Leuven, Leuven, Belgium adhesions using a modified Delphi method among an international panel of experts in an attempt to increase consistent outcome reporting in the field of intra-abdominal mesh-related adhesions [13]. Consistent outcome reporting provides us information on similarities and differences in both experimental and clinical settings and eventually allows for a correlation with clinically relevant outcomes.

\section{Material and methods}

The Delphi method is a technique to evaluate a topic and to try to reach consensus on this topic. It comprises of multiple rounds of questionnaires, which are completed individually by an expert panel. The Delphi method is characterized by anonymity, controlled feedback to the expert panel and statistical analysis of the results [14]. This study used the RAND-UCLA Appropriateness Method (RAM), which is a modified Delphi score specifically aimed at the medical field [15].

\section{Systematic review and panel formation}

PubMed, MEDLINE and EMBASE were searched to identify all publications on adhesion scoring models following the same methodology as recently published [16]. Search criteria included abdominal 'hernia/herniation/wall defect,' 'surgical mesh/meshes' and 'surgical adhesion/ tissue adhesion.' The search was limited to articles published between January 1, 1990, and December 31, 2015. English, Dutch and German were used as language restriction. Studies were included if they reported about the presence of a mesh, mesh-tissue adhesions and the use of an adhesion score. In vitro studies, literature reviews and letters to the editor were excluded.

The potential experts were identified as having published at least three full-text publications on adhesion scoring systems or complications due to mesh-related adhesions. After a first invitation by e-mail, two personalized reminders were sent after one and two weeks. In case authors did not respond to the reminders, they were considered unreachable and were not included in the expert panel.

\section{Questionnaires}

Questionnaires were designed and distributed online (SurveyMonkey Inc, Palo Alto, CA). The questionnaires addressed multiple choice questions regarding seven main topics: (1) utility of adhesion scoring models, (2) appropriateness of macroscopic variables, (3) appropriateness of microscopic variables, (4) composite scores versus 
subscores, (5) the range of scoring scales, (6) adhesionrelated complications and (7) follow-up length.

Panel members were able to rate the questions on a ninepoint Likert scale, ranging from very inappropriate (score 1) to very appropriate (score 9). Participants were encouraged to provide additional remarks in a free text field, such as other important variables or feedback on their answers.

At the end of each round, the results were statistically analyzed and presented during the follow-up round.

In follow-up rounds, items without consensus were repeated and adjusted if required, according to comments received in the previous round. Supplementary questions were developed and added to the follow-up rounds in order to establish concrete variables for a new adhesion score.

\section{Final consensus meeting}

The last round of the Delphi method was a face-to-face meeting held during the $38^{\text {th }}$ International Congress of the European Hernia Society (EHS) in Rotterdam, the Netherlands. During this meeting, all remaining items without consensus were discussed. All experts who finished two questionnaire rounds were invited to participate in the meeting. After the meeting, a summary of the discussion was sent to the entire panel, followed by two suggestions for a new adhesion scoring model. The final adhesion score was selected based on the expert panel preference.

\section{Statistical analysis}

All data were analyzed using MS Excel 2015 (Microsoft Corporation, Redmond WA, USA). Consensus was reached if the panel rated a topic unanimously as either inappropriate (panel median 1-3) or appropriate (panel median 7-9), without disagreement. Disagreement was tested to identify considerable dispersion between ratings of panel members, using the inter-percentile range adjusted for symmetry, according to the RAND-UCLA Appropriateness Method Manual and as used by Moossdorff et al. [15, 17].

\section{Results}

Fifty-seven international experts in the field of adhesion research were invited to participate, and 28 experts accepted the invitation to participate in this Delphi. Panel characteristics are shown in Table 1.

The first questionnaire was completed by 23 experts (82\%); 18 experts (64\%) completed the follow-up questionnaire. Ten of the 18 experts that finished the second round were able to also participate in the final face-to-face meeting. All 18 experts approved the last proposal of the new adhesion score. Figure 1 and Table 2 show a summary of the study outcomes and of the outcomes of the questionnaires and the face-to-face meeting.

\section{Questionnaires}

The first questionnaire consisted of 64 items distributed over seven categories. After the first round, consensus was reached on 44 out of 64 items $(69 \%)$ of the presented items. Disagreement existed on the remaining 20 items. Fourteen of these 20 items were rephrased into 22 separate items. Six items remained unchanged.

The second questionnaire consisted of the 28 items extracted from the first questionnaire, combined with 48 new items, leading to a total of 76 items. The 48 new items were added to address the formulation of a new adhesion score. At the end of the second round, consensus was reached on another 34 out of 76 items $(45 \%)$ of the presented items. Consensus was achieved on one out of six of the remaining, unchanged items and on eight out of 22 of the adjusted items from the first round (32\%). Regarding the new items, consensus was achieved on 25 out of 48 items $(52 \%)$.

\section{Final face-to-face meeting}

The main goal of the meeting was to formulate the final variables that had to be included in a new adhesion scoring model. Furthermore, the most appropriate scoring scales and follow-up times had to be selected. Feedback on the first two rounds was provided to the participants before the

Table 1 Characteristics of expert panel

\begin{tabular}{lr}
\hline Characteristics of expert panel & \\
\hline Gender & 13 \\
Male & 5 \\
Female & \\
Function & 5 \\
M.D./resident/surgeon & 2 \\
Postdoctoral researcher & 11 \\
Professor & \\
Country & 3 \\
Austria & 5 \\
Belgium & 1 \\
Germany & 2 \\
Spain & 4 \\
The Netherlands & 1 \\
Turkey & 2 \\
USA & 2 \\
\hline
\end{tabular}




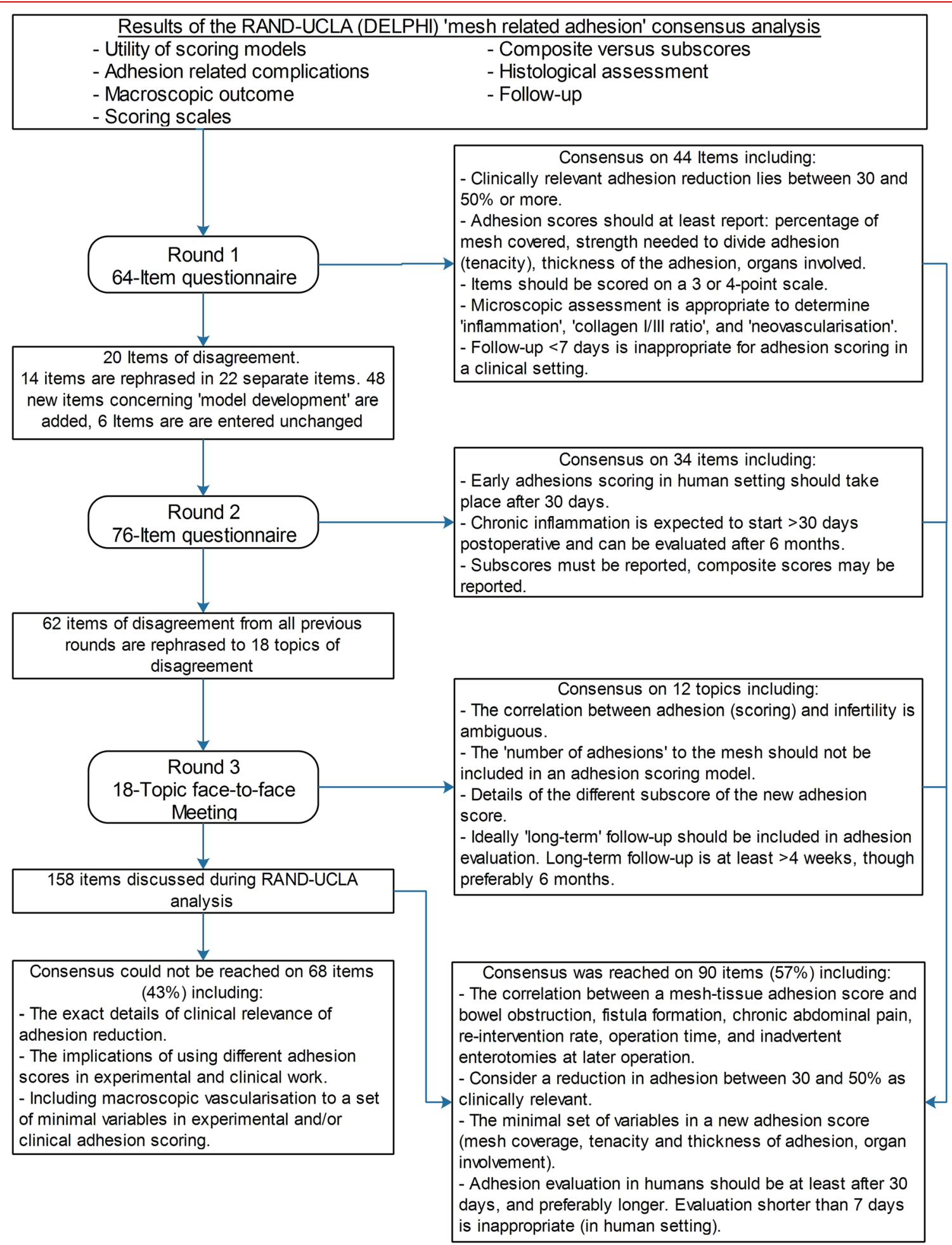

Fig. 1 Flow of information through the Delphi analysis. The Delphi analysis consisted of three rounds and resulted in a consensus on 57\% of the topics discussed. The figure provides a flowchart of information throughout the Delphi analysis, combined with the most important consensus reached in each round 
Table 2 Summary of outcomes of questionnaires and face-to-face meeting

\begin{tabular}{lcc}
\hline & Number of questions, $n$ & Consensus, $n(\%)$ \\
\hline Utility of adhesion scoring models & 10 & $7(70 \%)$ \\
Appropriateness of macroscopic variables & 42 & $19(45 \%)$ \\
Appropriateness of microscopic variables & 19 & $13(68 \%)$ \\
Composite scores versus subscores & 4 & $2(50 \%)$ \\
Range of scoring scales & 33 & $21(64 \%)$ \\
Adhesion-related complications & 9 & $7(78 \%)$ \\
Follow-up length & 41 & $24(59 \%)$ \\
\hline
\end{tabular}

final meeting. During the meeting, items on which no consensus was reached (summarized in 18 topics) were discussed. On 12 out of $18(66.7 \%)$ topics consensus was reached.

Next, a foundation for the scoring system was created and the scoring system was largely outlined. However, it was not possible to develop the scoring system to the smallest detail. Therefore, a subgroup of six authors drafted two detailed META scoring systems, based on the gathered information. These two proposals for the MEsh Tissue Adhesion (META) scoring system were developed and sent to the 18 experts from round two to review the newly developed scores and to express the preferred score. Consensus for one score was reached, although disagreement persisted. After adjusting the score a final version was sent to all the participants and approved.

\section{Consensus-based adhesion scoring model}

All experts consented that mesh-tissue adhesions should be scored systematically in both human and experimental settings, and that a uniform consensus-based scoring method should be used to increase interstudy comparability. According to the experts, mesh-related adhesion scores had to exist primarily of macroscopic variables. Variables included in the META scoring system are (1) percentage of mesh surface covered with adhesions, (2) strength needed to divide adhesions (tenacity), (3) thickness of adhesions and (4) organ involvement (see Table 3).

Any new scoring system for intra-abdominal mesh-tissue adhesions is preferably based on a four- or five-point scale to allow adequate variation in responses and statistical analyses. Follow-up times in experimental research should be at least 4 weeks after mesh implantation to evaluate chronic inflammation. Follow-up of clinical studies on mesh-related adhesions should include a minimum follow-up of 6 months.

\section{Discussion}

This paper describes the establishment of a consensusbased scoring system for mesh-related adhesions. The authors of this article hope that this score will be able to serve as a reporting standard for future research.

As such, the described score should be reported whenever adhesions with meshes in an experimental or human setting are studied. By doing so, we will be able to improve comparability between studies, reduce unnecessary experiments and hopefully correlate scores with clinical outcomes. Based on such research further refinement and validation of the scoring system will also be possible.

To validate the META scoring system, a small group of researchers, preferably from different research groups, should be formed. This group should score adhesions using the META scoring system, and subsequently, the outcomes should be compared. Ideally, adhesions are scored by researchers anonymously in an experimental setting. However, it might be hard to schedule an experiment at one time point with researchers from different international research groups and therefore a first step could be to score adhesions based on photographs. Next the scores should be compared, and differences in outcomes should be discussed. If it turns out that a description of one or more variables is unclear, the description will be adjusted. Subsequently, the research group should score the adhesions again, using this new description. Eventually, a meeting with the researchers should be scheduled to confirm the first outcomes in an experimental setting. Next, to validate the META scoring system, another group of researchers should approve the findings by performing the same experiment as described above.

It may seem unusual that yet another scoring system had to be determined, while over 20 adhesion scores already exist. However, a general disadvantage of these scores is that none of them have ever been validated, nor consistently used or reported by other research groups. Furthermore, variables in the adhesion scores have often been ill 
Table 3 META-consensus scoring system with each item scored and reported separately

\begin{tabular}{|c|c|}
\hline Item & Sco \\
\hline \multicolumn{2}{|l|}{ Percentage of mesh surface covered with adhesions } \\
\hline $0 \%$ & 0 \\
\hline $1-25 \%$ & 1 \\
\hline $26-50 \%$ & 2 \\
\hline $51-75 \%$ & 3 \\
\hline $76-100 \%$ & 4 \\
\hline \multicolumn{2}{|l|}{ Tenacity } \\
\hline No adhesions & 0 \\
\hline Loose adhesions easily released by traction only & 1 \\
\hline Adhesions require sharp dissection, no organ/serosal damage & 2 \\
\hline Adhesions require sharp dissection, with unavoidable organ/serosal damage & 3 \\
\hline \multicolumn{2}{|l|}{ Thickness of adhesions } \\
\hline No adhesions & 0 \\
\hline \multicolumn{2}{|l|}{ Single thin, filmy adhesion } \\
\hline \multicolumn{2}{|l|}{ Multiple thin, filmy adhesions } \\
\hline \multicolumn{2}{|l|}{ Single dense adhesion with or without filmy adhesions } \\
\hline Multiple dense adhesions with or without filmy adhesions & 4 \\
\hline \multicolumn{2}{|l|}{ Organ involvement } \\
\hline No adhesions & 0 \\
\hline \multicolumn{2}{|l|}{ Adhesions between mesh and omentum or a solid organ } \\
\hline Adhesions between mesh and part(s) of the intestinal tract & 2 \\
\hline Adhesions between mesh and part(s) of the intestinal tract with enteric fistul & 3 \\
\hline
\end{tabular}

The META-consensus score consists of four main items. (1) 'Percentage of mesh surface covered with adhesion' can either be scored by automated computer analysis of pictures taken during surgery or be estimated by the surgeon. (2) 'Tenacity' depends on the tools needed for adhesiolysis and relates to the strength of the adhesions. (3) 'Thickness of adhesions' is divided in four categories of adhesions. Macroscopically, two types of adhesions can be scored (filmy or dense) the categories are based on either single or multiple adhesions of both types. (4) 'Organ involvement' scores the adhered structures and increases in severity with increased adherence to the intestinal tract. The most severe category describes adhesions to the intestinal tract combined with macroscopic damage or interference with an organ such as fistula formation or bowel erosion

When reporting the META-consensus score, all items must be scored and reported individually in order to increase comparability between publications. An overall score can be calculated by adding all individual categories, though the relevance of this sum is unclear

Follow-up times in experimental research have to be a minimum of 4 weeks after mesh implantation. In clinical studies the minimal follow-up time should be 6 months

defined, making it difficult to replicate and interpret the scores correctly and therefore lead to a moderate reproducibility [18]. Even if the variables are clearly stated, the risk on limited reproducibility persists $[9,19]$. In order to create more uniformity and to increase reproducibility, the META scoring system has been developed based on the opinion of eighteen leading experts in the field of adhesion research, using a modified Delphi analysis. This analysis has proven to be of benefit, because it is developed for the medical field as a tool to combine best available evidence and experts opinion, when randomized clinical trials regarding a specific topic are absent [15]. Although the included variables do not differ much from the currently existing scores, the usage of the score and how researchers can assign a score to each variable have been extensively described.

It is recommended to report all variables separately to compare results between studies accurately and to enable future analyses of each variable. In such a way, it might lead to the conclusion that some variables closely correlate with one another and thus do not bring additional information, but even more so a correlation of one or more variables with clinically relevant outcomes could be examined more closely. The META scoring system score can be interpreted as a minimal set of outcome criteria that can be used as a standalone scoring system or be supplemented with other outcome variables, if desired.

The most important difference with other adhesion scores is that all authors realize that this scoring system is 
probably just a first step in order to create uniformity, hopefully leading to better understanding of mesh-tissue formation and important involved variables. With this information it could be that the current META scoring system requires adjustments. This awareness in combination with the desire to understand tissue adhesions and create uniformity in mesh-tissue adhesion research is the main strength of the META scoring system and hopefully contributes to the development of an ideal adhesion score, based on the data gathered using the META scoring system.

Another strength is that questionnaires were filled out anonymously, making experts' status influencing the outcomes impossible, thus leading to objective opinions. Unfortunately, not all invited researchers responded to our invitation to participate in this search for consensus. Authors who did become a panel member are convinced that a uniform scoring method is needed.

Next, some limitations must be addressed. The major weakness of the META scoring system and all other adhesion scores, is that the correlation between included variables and clinical outcomes is not clear yet. Ideally, higher scores correlate perfectly with impaired clinical outcomes. However, it is difficult to assess clinical outcomes, such as functional impairment, especially in experimental research, and therefore the currently included variables have been chosen as objectively measurable proxies. In the end, it may eventually appear that different scores for experimental and clinical setting should be used.

Next, ambiguity exists about topics as difference in tenacity and thickness of adhesions or follow-up time as can be explained by a lack of objective criteria and definitions. Since full consensus was not to be expected, the current consensus has attempted to describe these variables as simple and clear as possible. Nevertheless, interobserver differences can still occur and further assessment of the intra- and interobserver variability, also among different laboratories, is warranted.

Furthermore, consensus was reached on only 59\% of topics. Regarding the first main topic "utility of adhesion scoring models' it remained unclear whether the same adhesion score can be used in experimental and clinical research, since it is unclear what the relevance is of the presence of adhesions, even more so in different species or at different follow-up lengths. However, by using a uniform mesh adhesion scoring system, data from both experimental and clinical studies can be collected in order to compare these data.

Interestingly, the expert panel concluded that only a reduction in an adhesion score of at least $50 \%$ should be considered relevant. No consensus was reached on a reduction in adhesion scores of $20-30 \%$, even though $20-30 \%$ reduction has often been considered to be a good result in experimental research. However, during a gynecology consensus meeting regarding postoperative adhesion prevention, it was suggested that also a single adhesion can cause severe complications [20]. This could lead to the conclusion that only the presence of adhesions is of interest. However, experts feel that a higher adhesion score indicates a higher probability that clinical events may arrive. Next, a higher adhesion score is associated with an increased risk of enterotomies [21]. Yet, only describing the presence of adhesions is too limited to compare future studies and relate the outcomes with clinical outcomes.

The experts in our panel agreed that chronic abdominal pain, bowel obstruction, fistula formation, re-intervention rate, operation time and inadvertent enterotomies at later operations are associated with adhesions which are findings from large observational studies [3, 21, 22]. Interestingly, a recently published meta-analysis shows that the relation between adhesions and chronic abdominal pain is debatable. Although it is shown that adhesiolysis reduced chronic abdominal pain on the short term, it remains unclear whether adhesiolysis is effective on the long term. Considering the short-term results, there seems to be a relation between the presence of adhesions and chronic abdominal pain [23]. Regarding infertility, the expert panel agreed that they found it inappropriate to correlate adhesions and infertility, because too many factors are involved in infertility.

Recommended follow-up times are at least 4 weeks in experimental animal studies and 6 months in clinical studies to first evaluate chronic inflammation. After 4 weeks of follow-up in experimental research, it is believed that the chronic inflammatory phase starts and significant changes in adhesion morphology are unlikely to happen hereafter [5, 24]. Since the appropriate time point for long-term follow-up depends partly on the size of the animal, no recommendations regarding long-term followup are formulated. For practical reasons, it is considered appropriate in a clinical setting to score mesh-tissue adhesions during eventual future re-operations, thus usually on the long term.

Next to macroscopic variables, also microscopic variables are of interest in mesh-tissue adhesion research. According to this Delphi analysis, the presence of inflammatory cells, collagen and neovascularization are important variables and can provide further information regarding biocompatibility of meshes [25, 26]. However, the expert panel preferred separate scores for macroscopic and microscopic variables and chose not to include the microscopic items in the current adhesion scoring system.

An important factor for the META scoring system to succeed is an adequate distribution of the score. Therefore, the META scoring system will be distributed among the research groups and cooperating research groups of the 
expert panel. Each expert can explain how the score should be used, and if questions arise, the experts can answer these questions. Next, the META scoring system will be sent to all 57 experts, who were initially invited to participate in this study, since they have shown their interest in this topic. Furthermore, we want to present the results of this manuscript and the new scoring system at several congresses, in particular at hernia-specific congresses. With this strategy, a broad audience will be reached.

\section{Conclusion}

The META scoring system is a consensus-based minimal set of outcome parameters for clinical and animal (anti)adhesive research. It was agreed by a wide array of experts in the field of mesh-related adhesions to be a scoring system that can increase study comparability between different research groups and different research models and improve translatability to clinical practice. Wide implementation is however key, and the support of the scoring system by the current group of authors will hopefully be followed by new research groups and journal editors alike.

Funding No grants or other financial support were received for this study.

\section{Compliance with ethical standards}

Conflict of interest SM declares conflicts of interest, since he has received speaker honorarium from Bard, Medtronic, Stryker, Storz and Ethicon. Furthermore, he is a member of the Advisory Board from Bard. However, this fact did not influence the content of this paper. CD declares conflicts of interest, since she received consulting fees from Colorade Therapeutics, TELA Bio, Ethicon, Musculoskeletal Transplant Foundation, Biom'Up, CR Bard and Surgical Innovation Associates. In addition, she has a patent on Bionanocomposite for tissue regeneration and soft tissue repair issued. However, these facts did not influence the content of this paper. $\mathrm{LH}$, EM, JB, VG, KL, AV, ER, FB, SG, EA, RF, JG, KC, RK, JL, UK, $\mathrm{MM}, \mathrm{AP}, \mathrm{MS}, \mathrm{NB}$ declare no conflict of interest that influences the content of this paper.

Ethical approval This article does not contain any studies with human participants performed by any of the authors.

\section{References}

1. Luijendijk RW, de Lange DC, Wauters CC et al (1996) Foreign material in postoperative adhesions. Ann Surg 223(3):242-248

2. Turza KC, Butler CE (2012) Adhesions and meshes: synthetic versus bioprosthetic. Plast Reconstr Surg 130(5 Suppl 2):206s213s. https://doi.org/10.1097/PRS.0b013e3182638d48
3. ten Broek RP, Issa Y, van Santbrink EJ et al (2013) Burden of adhesions in abdominal and pelvic surgery: systematic review and met-analysis. BMJ 347:f5588. https://doi.org/10.1136/bmj. f5588

4. Zhu LM, Schuster P, Klinge U (2015) Mesh implants: an overview of crucial mesh parameters. World J Gastrointest Surg 7(10):226-236. https://doi.org/10.4240/wjgs.v7.i10.226

5. Schreinemacher MH, van Barneveld KW, Dikmans RE et al (2013) Coated meshes for hernia repair provide comparable intraperitoneal adhesion prevention. Surg Endosc 27(11):4202-4209. https://doi.org/10.1007/s00464-013-3021-5

6. Peeters E, van Barneveld KW, Schreinemacher MH et al (2013) One-year outcome of biological and synthetic bioabsorbable meshes for augmentation of large abdominal wall defects in a rabbit model. J Surg Res 180(2):274-283. https://doi.org/10. 1016/j.jss.2013.01.025

7. Gruber-Blum S, Brand J, Keibl C et al (2015) The impact of hydrophobic hernia mesh coating by omega fatty acid on atraumatic fibrin sealant fixation. Hernia J Hernias Abdom Wall Surg 19(4):651-657. https://doi.org/10.1007/s10029-014-1304-y

8. Diamond MP, Linsky CB, Cunningham T et al (1987) A model for sidewall adhesions in the rabbit: reduction by an absorbable barrier. Microsurgery 8(4):197-200

9. de Oliveira N, Paschoa AF, Crespo CC et al (1981) Use of the combination of a nitrofurazone derivative with polyethylene glycols in the prevention of the formation of peritoneal adhesions-experimental study. Arq Gastroenterol 18(2):54-59

10. Zuhlke HV, Lorenz EM, Straub EM et al (1990) Pathophysiology and classification of adhesions. Langenbecks Archiv fur Chirurgie Supplement II, Verhandlungen der Deutschen Gesellschaft fur Chirurgie Deutsche Gesellschaft fur Chirurgie Kongress, pp 1009-1016

11. Dubcenco E, Assumpcao L, Dray X et al (2009) Adhesion formation after peritoneoscopy with liver biopsy in a survival porcine model: comparison of laparotomy, laparoscopy, and transgastric natural orifice transluminal endoscopic surgery (NOTES). Endoscopy 41(11):971-978. https://doi.org/10.1055/s0029-1215229

12. Claudio RH, Diogo Filho A, Mamede Filho DO (2006) Peritoneostomy with latex coated polypropylene: experimental study in rats. Acta cirurgica brasileira/Sociedade Brasileira para Desenvolvimento Pesquisa em Cirurgia 21(6):402-408

13. Schreinemacher M, Henatsch D, van Barneveld K et al (2010) The need for standardised animal models and scoring systems in assessing mesh biocompatibility. Hernia J Hernias Abdom Wall Surg 14(3):335-336. https://doi.org/10.1007/s10029-010-0642-7

14. Dalkey NC (1967) Delphi. RAND Corporation, Santa Monica

15. Fitch K, Bernstein SJ, Aguilar MD et al (2001) The RAND/ UCLA appropriateness method user's manual. RAND Corporation, Santa Monica

16. Vogels RRM, Kaufmann R, van den Hil LCL et al (2017) Critical overview of all available animal models for abdominal wall hernia research. Hernia $J$ Hernias Abdom Wall Surg 21(5):667-675. https://doi.org/10.1007/s10029-017-1605-z

17. Moossdorff M, van Roozendaal LM, Strobbe LJ et al (2014) Maastricht Delphi consensus on event definitions for classification of recurrence in breast cancer research. J Natl Cancer Inst. https://doi.org/10.1093/jnci/dju288

18. Bellon JM, Contreras LA, Bujan J et al (1996) Effect of phosphatidylcholine on the process of peritoneal adhesion following implantation of a polypropylene mesh prosthesis. Biomaterials 17(14):1369-1372

19. Garrard CL, Clements RH, Nanney L et al (1999) Adhesion formation is reduced after laparoscopic surgery. Surg Endosc 13(1):10-13. https://doi.org/10.1007/s004649900887 
20. Diamond MP, Wexner SD, diZereg GS et al (2010) Adhesion prevention and reduction: current status and future recommendations of a multinational interdisciplinary consensus conference. Surg Innov 17(3):183-188. https://doi.org/10.1177/ 1553350610379869

21. ten Broek RP, Strik C, Issa Y et al (2013) Adhesiolysis-related morbidity in abdominal surgery. Ann Surg 258(1):98-106. https://doi.org/10.1097/SLA.0b013e31826f4969

22. Losanoff JE, Richman BW, Jones JW (2002) Entero-colocutaneous fistula: a late consequence of polypropylene mesh abdominal wall repair: case report and review of the literature. Hernia J Hernias Abdom Wall Surg 6(3):144-147. https://doi.org/ 10.1007/s10029-002-0067-z

23. van den Beukel BA, de Ree R, van Leuven S et al (2017) Surgical treatment of adhesion-related chronic abdominal and pelvic pain after gynaecological and general surgery: a systematic review and meta-analysis. Hum Reprod Update 23(3):276-288. https://doi. org/10.1093/humupd/dmx004
24. Matthews BD, Mostafa G, Carbonell AM et al (2005) Evaluation of adhesion formation and host tissue response to intra-abdominal polytetrafluoroethylene mesh and composite prosthetic mesh. J Surg Res 123(2):227-234. https://doi.org/10.1016/j.jss.2004.08. 012

25. Anderson JM, Rodriguez A, Chang DT (2008) Foreign body reaction to biomaterials. Semin Immunol 20(2):86-100. https:// doi.org/10.1016/j.smim.2007.11.004

26. Gomez-Gil V, Garcia-Honduvilla N, Pascual G et al (2010) Peritoneal adhesion formation and reformation tracked by sequential laparoscopy: optimizing the time point for adhesiolysis. Surgery 147(3):378-391. https://doi.org/10.1016/j.surg.2009. 10.011

Publisher's Note Springer Nature remains neutral with regard to jurisdictional claims in published maps and institutional affiliations. 